\title{
An Efficient Zero-Stable Numerical Method for Fourth-Order Differential Equations
}

\author{
S. J. Kayode \\ Department of Mathematical Sciences, School of Sciences, Federal University of Technology, PMB 704, \\ Akure, Ondo State, Nigeria
}

Correspondence should be addressed to S. J. Kayode, cosjkay57@yahoo.com

Received 9 July 2007; Revised 26 February 2008; Accepted 9 April 2008

Recommended by Michael Evans

The purpose of this paper is to produce an efficient zero-stable numerical method with the same order of accuracy as that of the main starting values (predictors) for direct solution of fourth-order differential equations without reducing it to a system of first-order equations. The method of collocation of the differential system arising from the approximate solution to the problem is adopted using the power series as a basis function. The method is consistent, symmetric, and of optimal order $p=6$. The main predictor for the method is also consistent, symmetric, zero-stable, and of optimal order $p=6$.

Copyright (C) 2008 S. J. Kayode. This is an open access article distributed under the Creative Commons Attribution License, which permits unrestricted use, distribution, and reproduction in any medium, provided the original work is properly cited.

\section{Introduction}

Many problems leading to fourth-order differential equations of the form

$$
y^{i v}=f\left(t, y, y^{1}, y^{\prime \prime}, y^{\prime \prime \prime}\right), \quad y^{(m-1)}\left(t_{0}\right)=\mu_{m-1}, \quad m=1,2,3,4,
$$

are of great interest to scientists and engineers formulating mathematical models for elastic bodies. Investigators, both theoretical and numerical analysts, have concerned themselves with the study and solutions of such equations. However, only a limited number of analytical methods are available for solving (1.1) directly without reduction to a first-order system of initial value problems (ivps) of type

$$
y^{\prime}=f(t, y), \quad y\left(t_{0}\right)=\mu, \quad f \in C^{1}[a, b], t \in R, y \in R^{m} .
$$

The many numerical methods adopted for such higher-order differential equations (1.1) are only for handling first-order equations (1.2), [1-4]. 
The reduction of such problems of type (1.1) to systems of first-order equations, to our knowledge, leads to serious computational burden as well as wastage in computer time. These setbacks had been addressed by some authors [5-7]. In recent times, attempts have been made to formulate numerical algorithms capable of solving problem (1.1) directly using differing methods of derivation with varying degree of accuracy [8-12]. These attempts are not without their associated limitations, some of which include low stepnumber $k$, too many function evaluations, and the combination of lower-order predictors with the correctors in the predictorcorrector methods $[13,14]$. All these have effects on the accuracy of the methods.

The proposed zero-stable order six methods are designed to have higher step number and reduced function evaluations, in order to address these observed problems. The derived method is capable of handling problems where $f$ is either linear or nonlinear. The numerical results of the method are compared with an existing method [3].

\section{Description of the method}

Let the approximate solution to problem (1.1) be a partial sum of a power series of the form:

$$
y(x)=\sum_{j=0}^{k+2} a_{j} x^{j}
$$

Taking the fourth derivative of (2.1) and using this in (1.1) yields

$$
\sum_{j=4}^{k+2} j(j-1)(j-2)(j-3) a_{j} x^{j-4}=f\left(x, y, y^{\prime}, y^{\prime \prime}, y^{\prime \prime \prime}\right) .
$$

To be able to obtain an open method with three function evaluations, collocation points are taken in (2.2) at all odd grid points $x=x_{n+i}, i=1(2) k$. Equation (1.2) is interpolated at all grid points $x=x_{n+i}, i=0(1) k-1, x \in R$, where $R$ is the set of real numbers. The choice of number of interpolation points was guided by the intended order of the method as well as the number of collocation points.

The system of equations obtained from the collocation and interpolation above is represented by the matrix equation:

$$
A X=B,
$$

where $A$, an $m$ by $m$ matrix, $X$ and $B$ are, respectively, given by

$$
A_{m \times m}=\left[\begin{array}{cccccccc}
0 & 0 & 0 & 0 & 24 & 120 x_{n+i} & \cdots & \xi x_{n+1}^{k-2} \\
0 & 0 & 0 & 0 & 24 & 120 x_{n+3} & \cdots & \xi x_{n+3}^{k-2} \\
\cdots & \cdots & \cdots & \cdots & \cdots & \cdots & \cdots & \cdots \\
0 & 0 & 0 & 0 & 24 & 120 x_{n+k} & \cdots & \xi x_{n+k}^{k-2} \\
1 & x_{n} & x_{n}^{2} & x_{n}^{3} & x_{n}^{4} & x_{n}^{5} & \cdots & x_{n}^{k+2} \\
1 & x_{n+1} & x_{n+1}^{2} & x_{n+1}^{3} & x_{n+1}^{4} & x_{n+1}^{5} & \cdots & x_{n+1}^{k+2} \\
\cdots & \cdots & \cdots & \cdots & \cdots & \cdots & \cdots & \cdots \\
\cdots & \cdots & \cdots & \cdots & \cdots & \cdots & \cdots & \cdots \\
1 & x_{n+k-1} & x_{n+k-1}^{2} & x_{n+k-1}^{3} & x_{n+k-1}^{4} & \cdots & \cdots & x_{n+k-1}^{k+2}
\end{array}\right],
$$




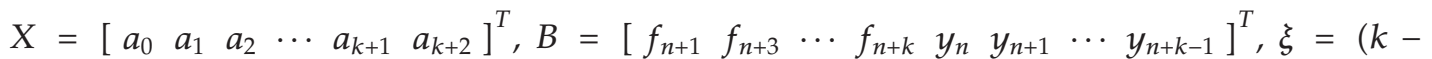
1) $k(k+1)(k+2), f_{n+i}=f\left(x_{n+i}, y_{n+i}, y_{n+i}^{\prime}, y_{n+i}^{\prime \prime}, y_{n+i}^{\prime \prime \prime}\right), i=0,1,2, \ldots, y_{n+i}=y\left(x_{n+i}\right)$, and $T$ is the usual matrix transpose.

Using the Gaussian method to solve the matrix (2.3), the values of $a_{j}^{\prime} s, j=0,1, \ldots, k+2$, for $k=5$, are obtained as shown in Appendix A. These values of $a_{j}^{\prime} s$ are substituted into (2.1). By using the transformation $x=t h+x_{n+4}, t \in(0,1]$ with $h$ being steplength, in the resulting equation after the substitution, an open method with variable coefficients is obtained as

$$
y(t)=\sum_{j=0}^{k-1} \alpha_{j}(t) y_{n+j}+h^{4} \sum_{j=0}^{k} \beta_{j}(t) f_{n+j}, \quad \beta_{k} \neq 0
$$

Taking $k=5$, the coefficients $\alpha_{j}(x)$ and $\beta_{j}(x)$ as well as their first, second, and third derivatives in (2.5) are computed and, respectively, obtained as shown in Appendix B.

The values of $t$ could be taken in the interval $I=(0,1]$ in order to obtain a particular discrete scheme. In this work, we take $t=1$ in (2.5) to have a zero-stable method and its derivatives as

$$
\begin{aligned}
& y_{n+5}=4 y_{n+4}-6 y_{n+3}+4 y_{n+2}-y_{n+1}+\frac{h^{4}}{24}\left(f_{n+5}+22 f_{n+3}+f_{n+1}\right), \\
& y_{n+5}^{\prime}=\frac{1}{210 h}\left\{853 y_{n+4}-1767 y_{n+3}+1128 y_{n+2}-157 y_{n+1}-57 y_{n}\right. \\
& \left.+\frac{h^{4}}{48}\left(1319 f_{n+5}+20738 f_{n+3}+1679 f_{n+1}\right)\right\}, \\
& y_{n+5}^{\prime \prime}=\frac{1}{60 h^{2}}\left\{119 y_{n+4}-236 y_{n+3}+54 y_{n+2}+124 y_{n+1}-60 y_{n}\right. \\
& \left.+\frac{h^{4}}{8}\left(159 f_{n+5}+1526 f_{n+3}+203 f_{n+1}\right)\right\}, \\
& y_{n+5}^{\prime \prime \prime}=\frac{1}{40 h^{3}}\left\{-23 y_{n+4}+132 y_{n+3}-258 y_{n+2}+212 y_{n+1}-63 y_{n}\right. \\
& \left.+\frac{h^{4}}{12}\left(317 f_{n+5}+1364 f_{n+3}+275 f_{n+1}\right)\right\}
\end{aligned}
$$

respectively.

The order of the open method (2.6) and its derivatives (2.7), (2.8), (2.9) is the same and is $p=6$. The error constant of (2.6) is found to be $C_{p+2} \approx-4.31 \times 10^{-2}$. The method is consistent and zero stable, satisfying the necessary and sufficient conditions for convergence of linear multistep methods $[3,4]$.

\section{Derivation of starting values for the method}

Method (2.6) above and its derivatives are open; $\beta_{k} \neq 0$. This implies that the sample discrete method (2.6) and its derivatives (2.7), (2.8), (2.9) require additional closed starting values, $\beta_{k}=0$, for the evaluation of $f_{n+i}, i=1,2, \ldots, k$. In this work, efforts are made at obtaining the main closed predictors of the same order of accuracy as starting values for $f_{n+i}, i=4, \ldots, k$. 
These predictors are obtained by using the same approach described in Section 2 . The following consistent and zero-stable closed methods are obtained, for $k=5,4$,

$$
y(t)=\sum_{j=0}^{k-1} \alpha_{j}(t) y_{n+j}+\sum_{j=0}^{k-1} \beta_{j}(t) f_{n+j}, \quad k=5,4
$$

Taking $t=1$ and for $k=5$, we have

$$
\begin{gathered}
y_{n+5}=4 y_{n+4}-6 y_{n+3}+4 y_{n+2}-y_{n+1}+\frac{h^{4}}{6}\left(f_{n+4}+4 f_{n+3}+f_{n+2}\right) \\
y_{N+5}^{\prime}=\frac{1}{210 h}\left\{898 y_{n+4}-1947 y_{n+3}+1398 y_{n+2}-337 y_{n+1}-12 y_{n}\right. \\
\left.\quad+\frac{h^{4}}{12}\left(159 f_{n+4}+132 f_{n+3}+115 f_{n+2}\right)\right\} \\
y_{n+5}^{\prime \prime}=\frac{1}{15 h^{2}}\left\{38 y_{n+4}-92 y_{n+3}+63 y_{n+2}-2 y_{n+1}-7 y_{n}\right. \\
\left.\quad+\frac{h^{4}}{8}\left(159 f_{n+4}+132 f_{n+3}+115 f_{n+2}\right)\right\} \\
y_{n+5}^{\prime \prime \prime}=-\frac{1}{10 h^{3}}\left\{\begin{array}{c}
11 y_{n+4}-54 y_{n+3}+96 y_{n+2}-74 y_{n+1}+21 y_{n} \\
+
\end{array}\right.
\end{gathered}
$$

The main predictors (3.2) and its associated derivatives (3.3), (3.4), and (3.5) are each of order 6 and their error constants $C_{p+2}$ are, respectively, $1.389 \times 10^{-3}, 6.948 \times 10^{-3}, 7.0367 \times 10^{-2}, 3.269 \times 10^{-1}$.

For $k=4$, we have the following:

$$
y_{n+4}=4 y_{n+3}-6 y_{n+2}+4 y_{n+1}-y_{n}+\frac{h^{4}}{6}\left(f_{n+3}+4 f_{n+2}+f_{n+1}\right),
$$

order $p=6, C_{p+2} \approx 1.389 \times 10^{-3}$;

$$
y_{n+4}^{(1)}=\frac{1}{6 h}\left\{26 y_{n+3}-57 y_{n+2}+42 y_{n+1}-11 y_{n}+\frac{h^{4}}{60}\left(185 f_{n+3}+452 f_{n+2}+113 f_{n+1}\right)\right\},
$$

order $p=5, C_{p+2} \approx 9.524 \times 10^{-3}$;

$$
y_{n+4}^{(2)}=\frac{1}{h^{2}}\left\{3 y_{n+3}-8 y_{n+2}+7 y_{n+1}-2 y_{n}+\frac{h^{4}}{60}\left(449 f_{n+3}+452 f_{n+2}+149 f_{n+1}\right)\right\},
$$

order $p=5, C_{p+2} \approx 7.778 \times 10^{-2}$;

$$
y_{n+4}^{(3)}=\frac{1}{h^{3}}\left\{y_{n+3}-3 y_{n+2}+3 y_{n+1}-y_{n}+\frac{h^{4}}{24}\left(55 f_{n+4} 8 f_{n+2}+13 f_{n+1}\right)\right\},
$$

order $p=5, C_{p+2} \approx 0.35$.

For $k<4$. 
To be able to have sufficient collocation and interpolation points at the grid points that will not produce system of underdetermined equations, the minimum stepnumber $k$ required for the derivation of any method for fourth-order problem is 4 . Therefore, Taylor series expansion was adopted to evaluate $y_{n+i}, y_{n+i}^{(1)}, y_{n+i}^{(2)}, y_{n+i}^{(3)}, i=1,2,3$; see Appendix C.

The initial values for the problems are used to evaluate $y_{0}, y_{0}^{(1)}, y_{0}^{(2)}, y_{0}^{(3)}$, where $y_{0}^{(r)}=$ $y^{(r)}\left(x_{0}\right), r=0,1,2,3$.

\section{Numerical experience}

The accuracy of the proposed method (2.6) has been tested, and the results of the following two initial value problems are shown in the tables in Appendix $\mathrm{C}$ below.

Test problem 1

$$
\begin{gathered}
y^{(4)}+y^{(2)}=0, \quad 0 \leq x \leq \frac{\pi}{2}, \quad y(0)=0, \\
y^{(1)}(0)=\frac{-1.1}{72-50 \Pi}, \quad y^{(2)}(0)=\frac{1}{144-100 \Pi}, \\
y^{(3)}(0)=\frac{1.2}{144-100 \Pi} . \\
\text { Theoretical solution: } y(x)=\frac{(1-x-\cos x-1.2 \sin x)}{(144-100 \pi)} .
\end{gathered}
$$

Test problem 2

$$
\begin{gathered}
y^{(4)}=\left(y^{(1)}\right)^{2}-y y^{(\#)}-4 x^{2}+e^{x}\left(1-4 x+x^{2}\right), \quad 0 \leq x \leq 1, \\
y(0)=1, \quad y^{(1)}(0)=1, \quad y^{(2)}(0)=3, \quad y^{(3)}(0)=1 .
\end{gathered}
$$

Theoretical solution: $y(x)=x^{2}+e^{x}$.

The test problems 1 and 2 were solved with method (2.6). The steplength $h=1 / 32$ is used for the purpose of comparison with the existing method Kayode [3]. The maximum errors obtained are compared with the method in Kayode [3] as shown in Tables 1 and 2.

\section{Conclusion}

A predictor-corrector method whose main predictors (3.2) and (3.6) have the same order of accuracy with the corrector (2.6) was formulated. The method was tested to be consistent and zero stable with order six. It is remarkable to note that the main predictors are of the same order 6 as that of the method with comparable error constants. This, to a large extent, reduces the effects that global error could have on the accuracy of the method.

The method was used to solve both linear and nonlinear problems of fourth-order differential equations without reduction to system of first order equations. 
Table 1: Comparison of errors in Kayode [3] and new method (2.6) for Problem 1.

\begin{tabular}{lllll}
\hline$X$ & $Y$-exact & $\begin{array}{l}Y \text {-computed with } \\
\text { new method }(2.6)\end{array}$ & $\begin{array}{l}\text { Errors in Kayode } \\
{[3]}\end{array}$ & $\begin{array}{l}\text { Errors in new } \\
\text { method }(2.6)\end{array}$ \\
\hline 0.103125 & $0.13007993 \mathrm{D}-02$ & $0.13007993 \mathrm{D}-02$ & $0.49873300 \mathrm{D}-15$ & $0.48355417 \mathrm{D}-16$ \\
0.206250 & $0.25317732 \mathrm{D}-02$ & $0.25317732 \mathrm{D}-02$ & $0.67654216 \mathrm{D}-15$ & $0.13933299 \mathrm{D}-15$ \\
0.306250 & $0.36524788 \mathrm{D}-02$ & $0.36524783 \mathrm{D}-02$ & $0.31350790 \mathrm{D}-14$ & $0.66893539 \mathrm{D}-15$ \\
0.406250 & $0.46959523 \mathrm{D}-02$ & $0.46959523 \mathrm{D}-02$ & $0.94360283 \mathrm{D}-14$ & $0.20129384 \mathrm{D}-14$ \\
0.506250 & $0.56576413 \mathrm{D}-02$ & $0.56576413 \mathrm{D}-02$ & $0.22116857 \mathrm{D}-13$ & $0.46736053 \mathrm{D}-14$ \\
0.603125 & $0.65077534 \mathrm{D}-02$ & $0.65077534 \mathrm{D}-02$ & $0.43379363 \mathrm{D}-13$ & $0.91874598 \mathrm{D}-14$ \\
0.703125 & $0.72983134 \mathrm{D}-02$ & $0.72983134 \mathrm{D}-02$ & $0.77870869 \mathrm{D}-13$ & $0.16069038 \mathrm{D}-13$ \\
0.803125 & $0.79985187 \mathrm{D}-02$ & $0.79985187 \mathrm{D}-02$ & $0.12863495 \mathrm{D}-12$ & $0.25407974 \mathrm{D}-13$ \\
0.903125 & $0.86072451 \mathrm{D}-02$ & $0.86072451 \mathrm{D}-02$ & $0.19927116 \mathrm{D}-12$ & $0.38108926 \mathrm{D}-13$ \\
1.003125 & $0.91242822 \mathrm{D}-02$ & $0.91242822 \mathrm{D}-02$ & $0.29323245 \mathrm{D}-12$ & $0.54051538 \mathrm{D}-13$ \\
\hline
\end{tabular}

Table 2: Comparison of errors in Kayode [3] and new method (2.6) for Problem 2.

\begin{tabular}{lllll}
\hline$X$ & $Y$-exact & $\begin{array}{l}Y \text {-computed with } \\
\text { new method }(2.6)\end{array}$ & $\begin{array}{l}\text { Errors in Kayode } \\
{[3]}\end{array}$ & $\begin{array}{l}\text { Errors in new } \\
\text { method }(2.6)\end{array}$ \\
\hline 0.103125 & $0.11192647 \mathrm{D}+01$ & $0.11192647 \mathrm{D}+01$ & $4.6842974 \mathrm{D}-11$ & $8.1714635 \mathrm{D}-12$ \\
0.206250 & $0.12715995 \mathrm{D}+01$ & $0.12715995 \mathrm{D}+01$ & $2.0687163 \mathrm{D}-10$ & $1.4680923 \mathrm{D}-10$ \\
0.306250 & $0.1452111 \mathrm{D}+01$ & $0.14521109 \mathrm{D}+01$ & $9.0421981 \mathrm{D}-10$ & $7.8813045 \mathrm{D}-10$ \\
0.406250 & $0.16662167 \mathrm{D}+01$ & $0.16662169 \mathrm{D}+01$ & $2.9137912 \mathrm{D}-09$ & $2.6795135 \mathrm{D}-09$ \\
0.506250 & $0.19153471 \mathrm{D}+01$ & $0.19153471 \mathrm{D}+01$ & $7.5114031 \mathrm{D}-09$ & $7.0615016 \mathrm{D}-09$ \\
0.603125 & $0.21915816 \mathrm{D}+01$ & $0.21915816 \mathrm{D}+01$ & $1.6231339 \mathrm{D}-08$ & $1.5438411 \mathrm{D}-08$ \\
0.703125 & $0.25144403 \mathrm{D}+01$ & $0.25144403 \mathrm{D}+01$ & $3.2237145 \mathrm{D}-08$ & $3.0900764 \mathrm{D}-08$ \\
0.803125 & $0.28775164 \mathrm{D}+01$ & $0.28775164 \mathrm{D}+01$ & $5.8891835 \mathrm{D}-08$ & $5.6759556 \mathrm{D}-08$ \\
0.903125 & $0.32829362 \mathrm{D}+01$ & $0.32829362 \mathrm{D}+01$ & $1.0079927 \mathrm{D}-07$ & $9.7549608 \mathrm{D}-08$ \\
1.003125 & $0.37330495 \mathrm{D}+01$ & $0.37330495 \mathrm{D}+01$ & $1.6373663 \mathrm{D}-07$ & $1.5897083 \mathrm{D}-07$ \\
\hline
\end{tabular}

The methods (3.2) and (3.6) developed as main predictors are of the same order of accuracy with the corrector (2.6). The method in Kayode [3] has predictors of lower order of accuracy, this accounts for an improvement of method (2.6) over that of [3] as compared in the maximum errors shown in Tables 1 and 2.

\section{Appendices}

\section{A. The values of $a_{j}^{\prime} s$}

$$
\begin{aligned}
a_{0}= & y_{n}-a_{1} x_{n}-a_{2} x_{n}^{2}-a_{3} x_{n}^{3}-a_{4} x_{n}^{4}-a_{5} x_{n}^{5}-a_{6} x_{n}^{6}-a_{7} x_{n}^{7} \\
a_{1}= & \frac{1}{h}\left(y_{n+1}-y_{n}\right)-a_{2}\left(2 x_{n}+h\right)-a_{3}\left(3 x_{n}^{2}+3 h x_{n}+h^{2}\right) \\
& -a_{4}\left(4 x_{n}^{3}+6 h x_{n}^{2}+4 h^{2} x_{n}+h^{3}\right)-a_{5}\left(5 x_{n}^{4}+10 h x_{n}^{3}+10 h^{2} x_{n}+5 h^{3} x_{n}+h^{4}\right) \\
& -a_{6}\left(6 x_{n}^{5}+15 h x_{n}^{4}+20 h^{2} x_{n}^{3}+15 h^{3} x_{n}^{2}+6 h^{4} x_{n}+h^{5}\right) \\
& -a_{7}\left(7 x_{n}^{6}+21 h x_{n}^{5}+35 h^{2} x_{n}^{4}+35 h^{3} x_{n}^{3}+21 h^{4} x_{n}^{2} 7 h^{5} x_{n}+h^{6}\right),
\end{aligned}
$$




$$
\begin{aligned}
a_{2}= & \frac{1}{2 h^{2}}\left(y_{n+2}-2 y_{n+1}+y_{n}\right)-a_{3}\left(3 x_{n}+3 h\right) \\
& -a_{4}\left(6 x_{n}^{2}+12 h x_{n}+7 h^{2}\right)-a_{5}\left(10 x_{n}^{3}+30 h x_{n}^{2}+35 h^{2} x_{n}+15 h^{3}\right) \\
& -a_{6}\left(15 x_{n}^{4}+60 h x_{n}^{3}+105 h^{2} x_{n}^{2}+90 h^{3} x_{n}+31 h^{4}\right) \\
& -a_{7}\left(21 x_{n}^{5}+105 h x_{n}^{4}+245 h^{2} x_{n}^{3}+315 h^{3} x_{n}^{2}+217 h^{4} x_{n}+63 h^{5}\right), \\
a_{3}= & \frac{1}{6 h^{3}}\left(3 y_{n+3}-3 y_{n+2} 3 y_{n+1}-y_{n}\right)-a_{4}\left(4 x_{n}+6 h\right) \\
& -a_{5}\left(10 x_{n}^{2}+30 h x_{n}+25 h^{2}\right)-a_{6}\left(20 x_{n}^{3}+90 h x_{n}^{2}+150 h^{2} x_{n}+90 h^{3}\right) \\
& -a_{7}\left(35 x_{n}^{4}+210 h x_{n}^{3}+525 h^{2} x_{n}^{2}+630 h^{3} x_{n}+301 h^{4}\right), \\
a_{4}= & \frac{f_{n+1}}{24}-5 a_{5}\left(x_{n}+h\right)-15 a_{6}\left(x_{n}^{2}+2 h x_{n}+h^{2}\right) \\
& -35 a_{7}\left(x_{n}^{3}+3 h x_{n}^{2}+3 h^{2} x_{n}+h^{3}\right), \\
a_{5}= & \frac{1}{240 h}\left(f_{n+3}-f_{n+1}\right)-6 a_{6}\left(x_{n}+2 h\right)-21 a_{7}\left(x_{n}^{2}+4 h x_{n}+\frac{91}{21} h^{2}\right), \\
a_{6}= & \frac{1}{2880 h}\left(f_{n+5}-2 f_{n+3}+f_{n+1}\right)-7 a_{6}\left(x_{n}+3 h\right), \\
a_{7}= & \frac{1}{20160 h}\left\{12\left(y_{n+4}-4 y_{n+3}+6 y_{n+2}-4 y_{n+1}+y_{n}\right)+h^{4}\left(f_{n+5}-8 f_{n+3}-5 f_{n+1}\right) .\right.
\end{aligned}
$$

\section{B. Coefficients $\alpha_{j}(x)$ and $\beta_{j}(x)$}

$$
\begin{aligned}
& \alpha_{0}(t)=\frac{1}{1680}\left(132 t+98 t^{2}-126 t^{3}-105 t^{4}-7 t^{5}+7 t^{6}+t^{7}\right), \\
& \alpha_{1}(t)=-\frac{1}{420}\left(272 t+308 t^{2}-56 t^{3}-105 t^{4}-7 t^{5}+7 t^{6}+t^{7}\right), \\
& \alpha_{2}(t)=\frac{1}{280}\left(552 t+658 t^{2}+14 t^{3}-105 t^{4}-7 t^{5}+7 t^{6}+t^{7}\right), \\
& \alpha_{3}(t)=-\frac{1}{420}\left(1392 t+1148 t^{2}+84 t^{3}-105 t^{4}-7 t^{5}+7 t^{6}+t^{7}\right), \\
& \alpha_{4}(t)=\frac{1}{1680}\left(1680+3212 t+1778 t^{2}+154 t^{3}-105 t^{4}-7 t^{5}+7 t^{6}+t^{7}\right), \\
& \beta_{1}(t)=\frac{1}{20160}\left(-240 t+284 t^{2}+630 t^{3}+420 t^{4}+35 t^{5}-28 t^{6}-5 t^{7}\right), \\
& \beta_{3}(t)=\frac{1}{10080}\left(1824 t+3920 t^{2}+2814 t^{3}+735 t^{4}-14 t^{5}-35 t^{6}-4 t^{7}\right), \\
& \beta_{5}(t)=\frac{1}{20160}\left(48 t+196 t^{2}+294 t^{3}+210 t^{4}+77 t^{5}+14 t^{6}+t^{7}\right) .
\end{aligned}
$$


First derivative of $\alpha_{j}(x)$ and $\beta_{j}(x)$ :

$$
\begin{aligned}
& \alpha_{0}^{\prime}(t)=\frac{1}{1680 h}\left(132+196 t-378 t^{2}-420 t^{3}-35 t^{4}+42 t^{5}+7 t^{6}\right), \\
& \alpha_{1}^{\prime}(t)=-\frac{1}{420 h}\left(272+616 t-168 t^{2}-420 t^{3}-35 t^{4}+42 t^{5}+7 t^{6}\right), \\
& \alpha_{2}^{\prime}(t)=\frac{1}{280 h}\left(552+1316 t+42 t^{2}-420 t^{3}-35 t^{4}+42 t^{5}+7 t^{6}\right), \\
& \alpha_{3}^{\prime}(t)=-\frac{1}{420 h}\left(1392+2296 t+252 t^{2}-420 t^{3}-35 t^{4}+42 t^{5}+7 t^{6}\right), \\
& \alpha_{4}^{\prime}(t)=\frac{1}{1680 h}\left(3212+3556 t+462 t^{2}-420 t^{3}-35 t^{4}+42 t^{5}+7 t^{6}\right), \\
& \beta_{1}^{\prime}(t)=\frac{1}{20160 h}\left(-240+56 t+1890 t^{2}+1680 t^{3}+175 t^{4}-168 t^{5}-35 t^{6}\right), \\
& \beta_{3}^{\prime}(t)=\frac{1}{10080 h}\left(1824+7820 t+8442 t^{2}+2940 t^{3}-70 t^{4}-210 t^{5}-28 t^{6}\right), \\
& \beta_{5}^{\prime}(t)=\frac{1}{20160 h}\left(48+392 t+882 t^{2}+840 t^{3}+385 t^{4}+84 t^{5}+7 t^{6}\right) .
\end{aligned}
$$

Second derivative of $\alpha_{j}(x)$ and $\beta_{j}(x)$ :

$$
\begin{aligned}
& \alpha_{0}^{\prime \prime}(t)=\frac{1}{1680 h^{2}}\left(196-756 t-1260 t^{2}-140 t^{3}+210 t^{4}+42 t^{5}\right), \\
& \alpha_{1}^{\prime \prime}(t)=-\frac{1}{420 h^{2}}\left(616-336 t-1260 t^{2}-140 t^{3}+210 t^{4}+42 t^{5}\right), \\
& \alpha_{2}^{\prime \prime}(t)=\frac{1}{280 h^{2}}\left(1316+84 t-1260 t^{2}-140 t^{3}+210 t^{4}+42 t^{5}\right), \\
& \alpha_{3}^{\prime \prime}(t)=-\frac{1}{420 h^{2}}\left(2296504 t-1260 t^{2}-140 t^{3}+210 t^{4}+42 t^{5}\right), \\
& \alpha_{4}^{\prime \prime}(t)=\frac{1}{1680 h^{2}}\left(3556924 t-1260 t^{2}-140 t^{3}+210 t^{4}+42 t^{5}\right), \\
& \beta_{1}^{\prime \prime}(t)=\frac{1}{20160 h^{2}}\left(56+3780 t+5040 t^{2}+700 t^{3}-840 t^{4}-210 t^{5}\right), \\
& \beta_{3}^{\prime \prime}(t)=\frac{1}{10080 h^{2}}\left(7820+16884 t+8820 t^{2}-280 t^{3}-1050 t^{4}-168 t^{5}\right), \\
& \beta_{5}^{\prime \prime}(t)=\frac{1}{20160 h^{2}}\left(392+1764 t+2520 t^{2}+1540 t^{3} 420 t^{4}+42 t^{5}\right) .
\end{aligned}
$$


Third derivative of $\alpha_{j}(x)$ and $\beta_{j}(x)$ :

$$
\begin{aligned}
& \alpha_{0}^{\prime \prime \prime}(t)=\frac{1}{1680 h^{3}}\left(-756-2520 t-420 t^{2}+840 t^{3}+210 t^{4}\right), \\
& \alpha_{1}^{\prime \prime \prime}(t)=-\frac{1}{420 h^{3}}\left(-336-2520 t-420 t^{2}+840 t^{3}+210 t^{4}\right), \\
& \alpha_{2}^{\prime \prime \prime}(t)=\frac{1}{280 h^{3}}\left(84-2520 t-420 t^{2}+840 t^{3}+210 t^{4}\right), \\
& \alpha_{3}^{\prime \prime \prime}(t)=-\frac{1}{420 h^{3}}\left(5044-2520 t-420 t^{2}+840 t^{3}+210 t^{4}\right), \\
& \alpha_{4}^{\prime \prime \prime}(t)=\frac{1}{1680 h^{3}}\left(924-2520 t-420 t^{2}+840 t^{3}+210 t^{4}\right), \\
& \beta_{1}^{\prime \prime \prime}(t)=\frac{1}{20160 h^{4}}\left(3780+10080 t+2100 t^{2}-3360 t^{3}-1050 t^{4}\right), \\
& \beta_{3}^{\prime \prime \prime}(t)=\frac{1}{10080 h^{4}}\left(16884+17640 t+2100 t^{2}-3360 t^{3}-1050 t^{4}\right), \\
& \beta_{5}^{\prime \prime \prime}(t)=\frac{1}{20160 h^{4}}\left(1764+5040 t+4620 t^{2}+1680 t^{3}+2100 t^{4}\right) .
\end{aligned}
$$

\section{Taylor series}

$$
\begin{aligned}
& y_{n+i} \equiv y\left(x_{n}+i h\right) \cong y\left(x_{n}\right)+i h y^{(1)}\left(x_{n}\right)+\frac{(i h)^{2}}{2 !} y^{(2)}\left(x_{n}\right)+\frac{(i h)^{3}}{3 !} y^{(3)}\left(x_{n}\right)+\frac{(i h)^{4}}{4 !} f_{n}+\frac{(i h)^{5}}{5 !} f_{n}^{(1)}+\cdots, \\
& y_{n+i}^{(1)} \equiv y^{(1)}\left(x_{n}+i h\right) \cong y^{(1)}\left(x_{n}\right)+i h y^{(2)}\left(x_{n}\right)+\frac{(i h)^{2}}{2 !} y^{(3)}\left(x_{n}\right)+\frac{(i h)^{3}}{3 !} f_{n}+\frac{(i h)^{4}}{4 !} f_{n}^{(1)}+\frac{(i h)^{5}}{5 !} f_{n}^{(2)}+\cdots, \\
& y_{n+i}^{(2)} \equiv y^{(2)}\left(x_{n}+i h\right) \cong y^{(2)}\left(x_{n}\right)+i h y^{(3)}\left(x_{n}\right)+\frac{(i h)^{2}}{2 !} f_{n}+\frac{(i h)^{3}}{3 !} f_{n}^{(1)}+\frac{(i h)^{4}}{4 !} f_{n}^{(2)}+\frac{(i h)^{5}}{5 !} f_{n}^{(3)}+\cdots, \\
& y_{n+i}^{(3)} \equiv y^{(3)}\left(x_{n}+i h\right) \cong y^{(3)}\left(x_{n}\right)+i h f+\frac{(i h)^{2}}{2 !} f_{n}^{(1)}+\frac{(i h)^{3}}{3 !} f_{n}^{(2)}+\frac{(i h)^{4}}{4 !} f_{n}^{(3)}+\cdots .
\end{aligned}
$$

Noting that in (1.1) $y^{(4)}\left(x_{n}\right)=f_{n}, y_{n}^{(4+j)}=f_{n}^{(j)}, j=0,1,2, \ldots, f_{n}^{(0)}=f_{n}$, and $f_{n}^{(j)}=f^{(j)}\left(x_{n}\right.$, $\left.y_{n}, y_{n}^{(1)}, y_{n}^{(2)}, y_{n}^{(3)}\right)$, the values of $f_{n}^{(1)}, f_{n}^{(2)}$ are obtained by partial derivative technique as

$$
\begin{aligned}
& \left.f^{(1)}=\frac{d f}{d x}=\frac{\partial f}{\partial x}+y^{(1)} \frac{\partial f}{\partial y}+y^{(2)} \frac{\partial f}{\partial y^{(1)}}+y^{(3)} \frac{\partial f}{\partial y^{(2)}}+f \frac{\partial f}{\partial y^{(3)}}\right), \\
& f^{(2)}=\frac{d^{2} f}{d x^{2}}=2\left(A y^{(1)}+B y^{(2)}+C y^{(3)}+D f\right)+E+F
\end{aligned}
$$


where

$$
\begin{gathered}
A=\frac{\partial^{2} f}{\partial x \partial y}+y^{(2)} \frac{\partial^{2} f}{\partial y \partial y^{(1)}}+y^{(3)} \frac{\partial^{2} f}{\partial y \partial y^{(2)}}+f \frac{\partial^{2} f}{\partial y \partial y^{(3)}} \\
B=\frac{\partial^{2} f}{\partial x \partial y}+y^{(3)} \frac{\partial^{2} f}{\partial y^{(1)} \partial y^{(2)}}+f \frac{\partial^{2} f}{\partial y^{(1)} \partial y^{(3)}}, \\
C=\frac{\partial^{2} f}{\partial x \partial y^{(2)}}+f \frac{\partial^{2} f}{\partial y^{(2)} \partial y^{(3)}}, \\
D=\frac{\partial^{2} f}{\partial x \partial y^{(3)}}, \\
E=y^{(2)} \frac{\partial f}{\partial y}+y^{(3)} \frac{\partial f}{\partial y^{(1)}}+f \frac{\partial f}{\partial y^{(2)}}+f^{\prime} \frac{\partial f}{\partial y^{(3)}}, \\
F=\frac{\partial^{2} f}{\partial x^{2}}+\left(y^{(1)}\right)^{2} \frac{\partial^{2} f}{\partial y^{2}}+\left(y^{(2)}\right)^{2} \frac{\partial^{2} f}{\left(\partial y^{(1)}\right)^{2}}+\left(y^{(3)}\right)^{2} \frac{\partial^{2} f}{\left(\partial y^{(2)}\right)}+(f)^{2} \frac{\partial^{2} f}{\left(\partial y^{(3)}\right)^{2}}
\end{gathered}
$$

\section{References}

[1] C. E. Abhulimen and F. O. Otunta, "A sixth order multiderivative multistep methods for stiff system of differential equations," International Journal of Numerical Mathematics, vol. 1, no. 2, pp. 248-268, 2006.

[2] R. P. K. Chan, P. Leone, and A. Tsai, "Order conditions and symmetry for two-step hybrid methods," International Journal of Computer Mathematics, vol. 81, no. 12, pp. 1519-1536, 2004.

[3] S. J. Kayode, "An order six zero-stable method for direct solution of fourth order ordinary differential equations," American Journal of Applied Sciences. In press.

[4] J. D. Lambert, Computational Methods in Ordinary Differential Equations, John Wiley \& Sons, London, UK, 1973.

[5] D. O. Awoyemi, "A new sixth-order algorithm for general second order ordinary differential equations," International Journal of Computer Mathematics, vol. 77, no. 1, pp. 117-124, 2001.

[6] D. O. Awoyemi, "A P-stable linear multistep method for solving general third order ordinary differential equations," International Journal of Computer Mathematics, vol. 80, no. 8, pp. 987-993, 2003.

[7] D. O. Awoyemi and S. J. Kayode, "An optimal order continuous multistep algorithm for initial value problems of special second order differential equations," Journal of the Nigerian Association of Mathematical Physics, vol. 6, pp. 285-292, 2002.

[8] R. A. Bun' and E. D. Vasil'ev, "A numerical method for solving differential equations with an arbitrary order of derivatives," Computational Mathematics and Mathematical Physics, vol. 32, no. 3, pp. 317-330, 1992.

[9] S. O. Fatunla, Numerical Methods for Initial Value Problems in Ordinary Differential Equations, Computer Science and Scientific Computing, Academic Press, Boston, Mass, USA, 1988.

[10] G. Pittaluga, L. Sacripante, and E. Venturino, “A collocation scheme for linear third order differential equations," Quaderno, N. 32, 2004.

[11] I. Jacques and C. Judd, Numerical Analysis, Chapman \& Hall, London, UK, 1987.

[12] S. J. Kayode and D. O. Awoyemi, "A 5-step maximal order method for direct solution of second order ordinary differential equations," Journal of the Nigerian Association of Mathematical Physics, vol. 9, pp. 279-284, 2005.

[13] D. O. Awoyemi, "An algorithmic collocation approach for direct solution of special and general fourth-order initial value problems of ordinary differential equations," Journal of the Nigerian Association of Mathematical Physics, vol. 6, pp. 271-284, 2002.

[14] D. O. Awoyemi and S. J. Kayode, "Maximal order multiderivative collocation method for direct solution of fourth order initial value problems of ordinary differential equations," Journal of the Nigerian Mathematical Society, vol. 23, pp. 53-64, 2004. 


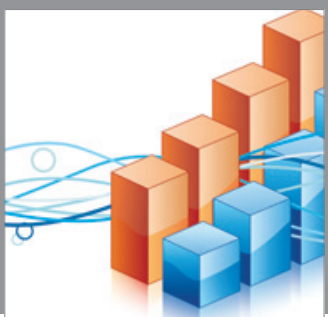

Advances in

Operations Research

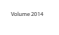

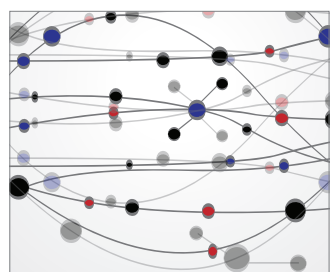

\section{The Scientific} World Journal
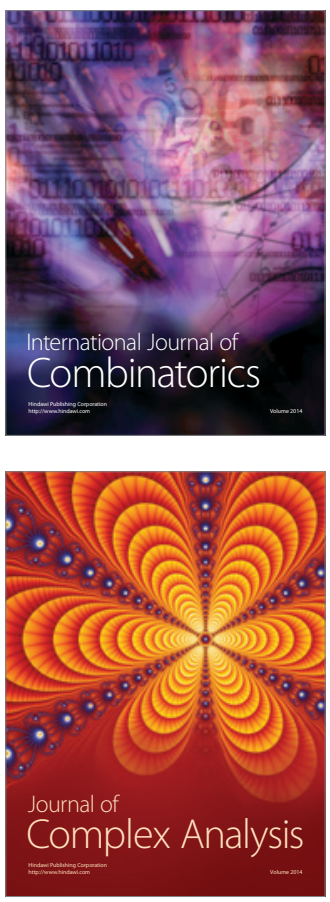

International Journal of

Mathematics and

Mathematical

Sciences
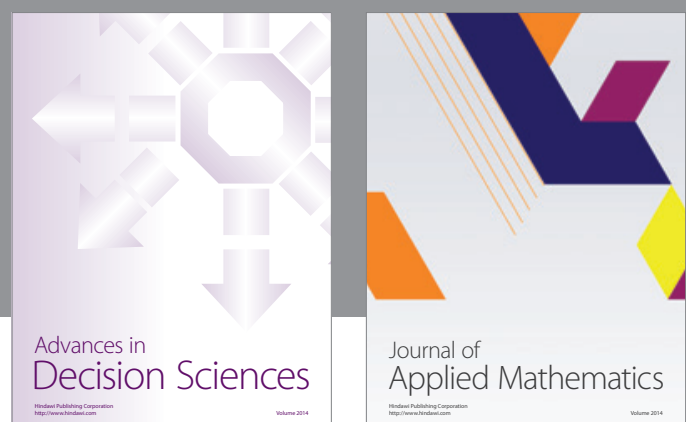

Journal of

Applied Mathematics
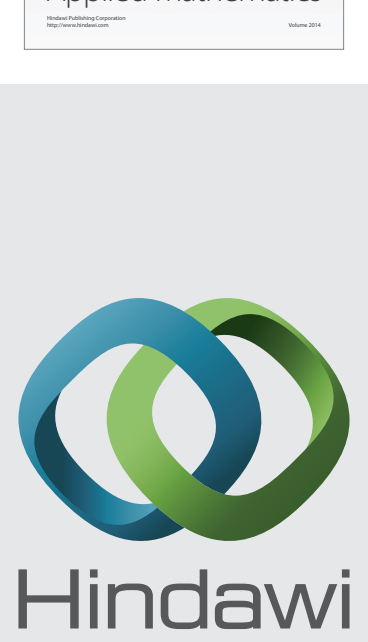

Submit your manuscripts at http://www.hindawi.com
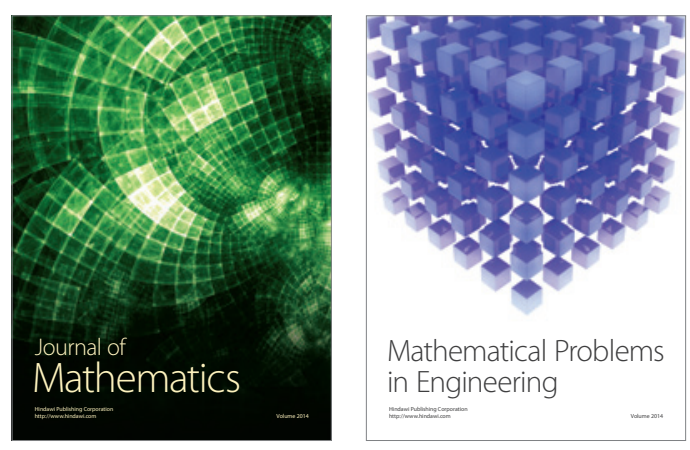

Mathematical Problems in Engineering
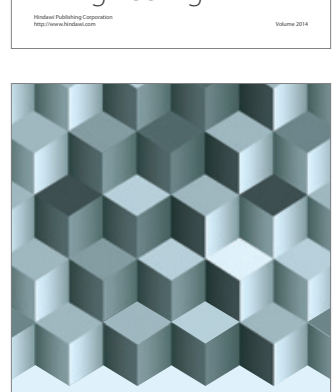

Journal of

Function Spaces
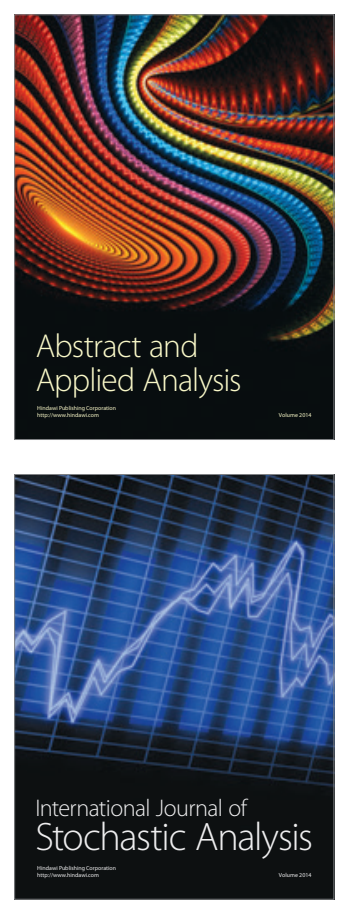

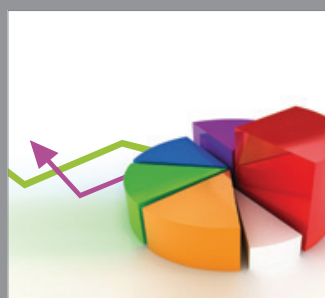

ournal of

Probability and Statistics

Promensencen
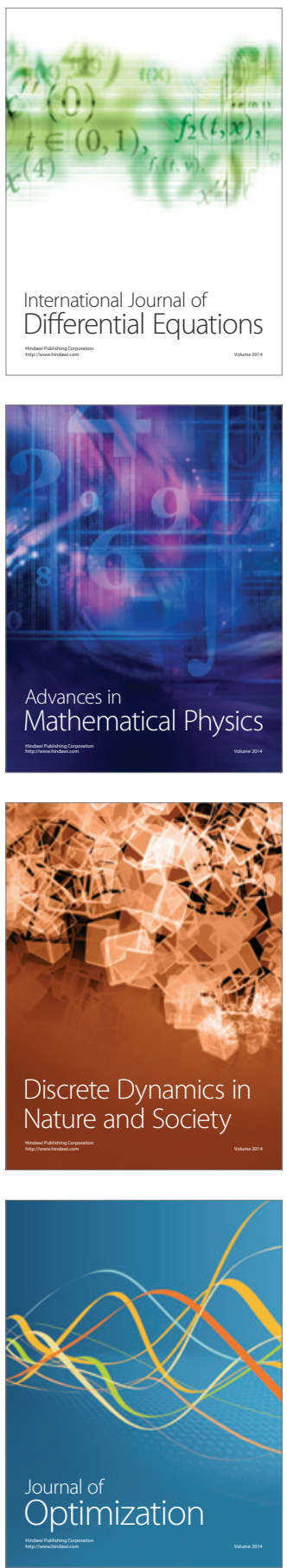